\title{
PRECISE CONTROL OF SMALL SATELLITES IN CLOSE CLUSTERS USING SIMPLIFIED NONLINEAR MODEL PREDICTIVE CONTROL
}

\author{
AJAY GAUTAM \\ School of Electrical, Electronics and Communication Engineering, Korea University of Technology and Education,
}

Cheonan, South Korea

\begin{abstract}
Precise translational control is explored with a simplified nonlinear model predictive control (MPC) scheme for member satellites in a close cluster in an Earth-orbiting environment. Recognizing that the nonlinear dynamical effects in relative satellite dynamics are small but complicated to be directly incorporated into the dynamical model used in the MPC formulation, we present a successive-optimization-based MPC scheme in which the nonlinear effects are incorporated in the form of constant disturbances which are evaluated and updated between two successive optimizations through an intervening high-fidelity nonlinear propagation of the member satellite orbit. The convergence of the predicted disturbance sequence with successive optimizations allows us to make accurate predictions about the future trajectory of the satellite and thus to precisely control its state. The effectiveness of the proposed scheme is evaluated through extensive nonlinear simulations.
\end{abstract}

KEYWORDS: Small satellite clusters, Satellite formations, Model predictive control (MPC) \& Nonlinear MPC

Received: Jun 05, 2020; Accepted: Jun 25, 2020; Published: Jul 04, 2020; Paper Id.: IJMPERDJUN2020200

\section{INTRODUCTION}

Clusters, formations or swarms of low-cost miniaturized spacecraft with distributed sensing and measurement capabilities have been explored for the past several years and they are envisaged to offer promising outcomes in applications involving the imaging of the Earth and the heavenly bodies, the collection of atmospheric and meteorological data and other scientific observations [1]. Several missions involving fleets of small satellites have been considered for various topographical, meteorological and astronomical applications that require space-based sensors with large aperture sizes (e.g., [2-5]). The motivation for the use of a fleet of small satellites instead of a single large satellite in such applications includes simple and low-cost design, increased redundancy, reconfigurability and mission flexibility [6].

In a formation or a swarm mission, a set of spatially distributed spacecraft need to move forward forming a particular pattern while maintaining a precise relative position, velocity, attitude and angular velocity with each other. Advanced coordination and control algorithms have been explored for various missions envisaged in the past and an early survey on the topic can be found in [6]. More challenging objectives have been considered in the recent years and much more has been envisaged for future missions [7]. Coordination and control in a formation or a swarm mission are aimed at configuring or reconfiguring a set of member satellites into a desired spatial pattern and maintaining the chosen pattern by utilizing limited control resources available in the member satellites. Depending on the way control-related information is interchanged among the members, various high-level coordination architectures such as leader-following, cyclic, consensus-based and so on have been explored for multi-satellite 
missions (see, e.g. [8-10]).

The remoteness of the location, the limitations in the available resources and the dynamic environment of the orbits make a multi-satellite application challenging [11-12]. In the Earth-orbiting environment, while many cluster patterns are theoretically maintainable under the ideal gravitational field, practically, member satellites need regular control to counter disturbing forces that make them drift apart from their desired spatial states forming the chosen pattern [6]. The problem of driving a satellite to a desired relative state has been addressed with various methods including traditional proportionalderivative, linear-quadratic (LQ), adaptive and so on for the past several years. Since the problem involves constraints of various kinds, several forms of model-predictive control (MPC) methods have been explored extensively in the recent years. (e.g., [13-16]). Papers such as [13] and [14] have employed robust forms of MPC that ensure stability and robustness in the presence of disturbances and unmodeled nonlinearities. In [15] and [16], forms of nonlinear MPC have been explored. While [15] has used an explicit offline solution of the MPC problem to reduce the online computational complexity, the authors in [16] have considered the problem dealing with larger relative distances for a mission along an elliptical orbit for which a nonlinear model is necessary.

While treating unmodeled nonlinearities and perturbations as disturbances with a robust control formulation likely makes the control effort conservative and reduces the control precision (see, e.g., [17]), the models for various nonlinear effects such as gravity harmonics including $J_{2}$, sun-moon effects etc. are complicated and not readily incorporable in the discrete-time dynamics model to be used in a control approach like MPC. In this paper, we explore a simplified nonlinear MPC approach for the precise control of satellites in a close cluster in order drive them to their desired translational states. We use a successive-optimization-based approach using a linear model with an intervening high-fidelity nonlinear propagation step so as to precisely incorporate the effects of deterministic perturbations in the prediction model. Such an approach was explored early on as a successive substitution approach in the context of dynamic matrix control of slow processes such as in polymerization reactors [18]. Much faster processing speeds of computing hardware now allow us to use the successive optimization approach with intervening nonlinear propagations over large horizon lengths at every time step. We assess and illustrate the effectiveness of the proposed MPC strategy with realistic simulation results using highfidelity nonlinear simulations.

\section{SYSTEM DESCRIPTION - SATELLITE RELATIVE DYNAMICS}

In a satellite cluster or formation, each satellite has to track its desired state expressed with respect to a reference point to maintain the chosen configuration or pattern. Generally, the equations that represent the dynamics of a satellite moving in a fleet are first developed in the earth-centered inertial (ECI) coordinate frame and then expressed in a local coordinate frame, also called local-vertical local-horizontal (LVLH) frame or Hill's frame [13,14], centered at the reference point and rotating around the earth with the fleet along a reference orbit. The fleet reference point is described by a virtual (or real) satellite moving along a reference orbit represented by a set of standard orbital elements $\Xi=(a, e, i, \Omega, \omega, \theta)$, where $a, e, i, \Omega, \omega$ and $\theta$ correspond respectively to the semimajor axis, eccentricity, inclination, right ascension of the ascending node, argument of the periapsis and true anomaly.

Although it is sometimes preferable to express absolute or relative dynamics of a member satellite in terms of its orbital elements or differential orbital elements, we often consider the relative dynamics w.r.t. the reference point in terms of the relative displacements $x, y$ and $z$ and velocities $\dot{x}, \dot{y}$ and $\dot{z}$ along the three standard directions of the local rotating frame, viz., the radial (along the radius vector $R_{r e f}$ ), along-track (along the direction of motion of the reference point) and 
cross-track (normal to the reference orbital plane) directions. The equations of the relative motion of a member satellite with respect to the reference point in a general elliptical orbit of eccentricity, $e$, are given by

$$
\begin{aligned}
& \ddot{x}=\frac{3+e \cos \theta}{1+e \cos \theta} w^{2} x-\frac{2 e \sin \theta}{1+e \cos \theta} w^{2} y+2 w \dot{y}+u_{x}+d_{x} \\
& \ddot{y}=\frac{2 e \sin \theta}{1+e \cos \theta} w^{2} x+\frac{e \cos \theta}{1+e \cos \theta} w^{2} y-2 w \dot{x}+u_{y}+d_{y} \\
& \ddot{z}=-\frac{w^{2}}{1+e \cos \theta} z+u_{z}+d_{z}
\end{aligned}
$$

where, $u_{x}, u_{y}, u_{z}$ denote the components of the control input and $d_{x}, d_{y}, d_{z}$ represent the components of the 'disturbance' acceleration which includes the effects of nonlinearities, perturbations, sun-moon effects and other external disturbances such as atmospheric drag and radiation pressure. In the equations, $\theta$ is the true anomaly and $w=\sqrt{\frac{\mu}{a^{3}}}(1+$ $e \cos \theta)^{2} /\left(1-e^{2}\right)^{3 / 2}$ is the angular velocity of the reference orbit. The true anomaly $\theta$ is monotonically related with the mean anomaly of the reference orbit, $M$ by the relationship $M=E-e \sin E$, where $E=2 \tan ^{-1}\left(\sqrt{\frac{1-e}{1+e}} \tan \frac{\theta}{2}\right)$. Further, $M$ is linearly related with time $t$ by the equation $t-t_{p}=\sqrt{\frac{a^{3}}{\mu}} M$ where $t_{p}$ is the time of periapsis passage.

Defining the state vector $X=\left[\begin{array}{llllll}x & y & z & \dot{x} & \dot{y} & \dot{z}\end{array}\right]^{T}$, the input vector $u=\left[\begin{array}{lll}u_{x} & u_{y} & u_{z}\end{array}\right]^{T}$, and the vector of disturbance effects $d=\left[\begin{array}{lll}d_{x} & d_{y} & d_{z}\end{array}\right]^{T}$, we write the relative motion dynamics as

$$
\dot{X}=A_{c} X+B_{c} u+B_{d c} d
$$

where matrix $A_{c}=\left[\begin{array}{cc}0_{3 \times 3} & I_{3} \\ A_{c 21} & A_{c 22}\end{array}\right], B_{d c}=B_{c}=\left[\begin{array}{c}0_{3 \times 3} \\ I_{3}\end{array}\right]$ with

$$
A_{c 21}=\frac{1}{1+e \cos \theta}\left[\begin{array}{ccc}
(3+e \cos \theta) w^{2} & -2 e(\sin \theta) w^{2} & 0 \\
2 e(\sin \theta) w^{2} & e(\cos \theta) w^{2} & 0 \\
0 & 0 & -w^{2}
\end{array}\right], \quad A_{c 22}=\left[\begin{array}{ccc}
0 & 2 w & 0 \\
-2 w & 0 & 0 \\
0 & 0 & 0
\end{array}\right]
$$

Since $\theta$ and $w$ are time-varying, system matrix $A_{c}$ in (1) is time-varying for any $e \neq 0$. For a circular reference orbit, $e=0$, and, if we neglect the nonlinearities represented by $d(t)$, system (1) becomes an LTI system

$$
\dot{X}=\bar{A}_{c} X+B_{c} u+B_{d c} d
$$

with a constant system matrix $\bar{A}_{c}=\left[\begin{array}{cc}0_{3 \times 3} & I_{3} \\ \bar{A}_{c 21} & \bar{A}_{c 22}\end{array}\right]$ with $\bar{A}_{c 21}=\left[\begin{array}{ccc}3 \bar{w}^{2} & 0 & 0 \\ 0 & 0 & 0 \\ 0 & 0 & -\bar{w}^{2}\end{array}\right], \bar{A}_{c 22}=\left[\begin{array}{ccc}0 & 2 \bar{w} & 0 \\ -2 \bar{w} & 0 & 0 \\ 0 & 0 & 0\end{array}\right]$.

where $\bar{w}=\sqrt{\frac{\mu}{a^{3}}}$ is the mean motion of the reference orbit. Equations in (2) are known as Hill-Clohessy-Wiltshire equations.

Several geometrical patterns considered for satellite fleets or formations are based on the unforced form of model (2), the solution of which gives periodic relative orbits that do not drift away from the reference point. These passive relative orbits form the fleet patterns that are naturally supported if we neglect the disturbance effects, and can be maintained for a long time. Member satellites can be placed in such orbits with particular choices of initialization conditions. 
Considering the disturbance vector $d$ to be unrelated with the state and the input, we can obtain an approximate discrete-time model of (1). For a circular reference orbit, we can obtain and use the discrete-time model by exactly discretizing (2) assuming that the disturbances remain constant over the sampling period:

$$
X_{k+1}=A X_{k}+B u_{k}+B_{d} d_{k}
$$

We consider reference orbits of small eccentricity in the sequel and intend to use the discrete-time model (3) in the MPC scheme. Since control inputs in satellites are usually impulsive, we consider only a fraction of the sampling time to be used in the application of control. Let $T$ be the sampling period and $T, 0<c<1$, be the duration in the beginning of the sampling period during which the control input is applied. Then, the discrete-time system matrices $A, B$ and $B_{d}$ are given by

$$
A=e^{A_{C} T}, \quad B=\int_{\tau=0}^{c T} e^{A_{C}(T-\tau)} B d \tau, \quad B_{d}=\int_{\tau=0}^{T} e^{A_{C}(T-\tau)} B_{d} d \tau
$$

Note that in model (3) $d_{k}$ mainly depends, in a nonlinear way, on $X_{k}$, $u_{k}$, orbital elements of the reference orbit and so on. While $d_{k}$ is small for a close cluster, its effects gradually grow and become significant over long periods of time, and hence need to be corrected by applying the control input. While we may obtain a satisfactory performance by treating $d_{k}$ as being bounded in a set, for precise controls with limited control resources, it is necessary to account for the actual or closeto-actual values of $d_{k}$.

\section{SIMPLIFIED MPC THROUGH NONLINEARITY-INCORPORATED SUCCESSIVE OPTIMIZATION}

MPC is a popular control technique widely used in the process industries and various other areas. It is based on a constrained online optimization, at each time step $k$, of control inputs for minimizing a predicted cost function over a finite horizon [19].

\section{Cost Function}

The standard quadratic cost function for the discrete-time system of (3) can be expressed as:

$$
\begin{gathered}
J_{N}(k)=\sum_{i=0}^{N-1}\left\{\left(X_{k+i \mid k}-\bar{X}_{k+i \mid k}\right)^{T} Q\left(X_{k+i \mid k}-\bar{X}_{k+i \mid k}\right)+\left(u_{k+i \mid k}-\bar{u}_{k+i \mid k}\right)^{T} R\left(u_{k+i \mid k}-\bar{u}_{k+i \mid k}\right)\right\} \\
+\left(X_{k+N \mid k}-\bar{X}_{k+N \mid k}\right)^{T} P\left(X_{k+N \mid k}-\bar{X}_{k+N \mid k}\right)
\end{gathered}
$$

where $Q$ and $P$ are positive semi-definite matrices and $R$ is a positive definite matrix. Here, $X_{k+i \mid N}$, and $u_{k+i \mid N}$ etc. denote the predicted values of the respective variables of time $k+i$ based on the prediction made at time $k$. For each time $k+i$, $\bar{X}_{k+i \mid k}$ and $\bar{u}_{k+i \mid k}$ form the operating point state-input pair, i.e., the desired value of the state-input pair for time $k+i$. In the satellite problem, we consider passive relative desired path $\bar{X}_{k+i \mid k}, k \geq 0$ for each satellite so that $\bar{u}_{k+i \mid k}=0, i \geq 0$. Applying the dynamics relationship (3) to the predicted states and inputs, we can write the cost function $J_{N}(k)$ in a compact form as

$$
J_{N}(k)=\left(\boldsymbol{A} X_{k}+\boldsymbol{B} \boldsymbol{u}_{k}+\boldsymbol{B}_{\boldsymbol{d}} \boldsymbol{d}_{k}-\overline{\boldsymbol{X}}_{k}\right)^{T} \boldsymbol{Q}\left(\mathcal{A} X_{k}+\boldsymbol{B} \boldsymbol{u}_{k}+\boldsymbol{B}_{\boldsymbol{d}} \boldsymbol{d}_{k}-\overline{\boldsymbol{X}}_{k}\right)+\left(\boldsymbol{u}_{k}-\overline{\boldsymbol{u}}_{k}\right)^{T} \mathcal{R}\left(\boldsymbol{u}_{k}-\overline{\boldsymbol{u}}_{k}\right)
$$

where 


$$
\overline{\boldsymbol{X}}_{k}=\left[\begin{array}{c}
\bar{X}_{k \mid k} \\
\bar{X}_{k+1 \mid k} \\
\bar{X}_{k+2 \mid k} \\
\vdots \\
\bar{X}_{k+N \mid k}
\end{array}\right], \quad \boldsymbol{u}_{k}=\left[\begin{array}{c}
u_{k \mid k} \\
u_{k+1 \mid k} \\
u_{k+2 \mid k} \\
\vdots \\
u_{k+N-1 \mid k}
\end{array}\right], \quad \overline{\boldsymbol{u}}_{k}=\left[\begin{array}{c}
\bar{u}_{k \mid k} \\
\bar{u}_{k+1 \mid k} \\
\bar{u}_{k+2 \mid k} \\
\vdots \\
\bar{u}_{k+N-1 \mid k}
\end{array}\right], \quad \boldsymbol{d}_{k}=\left[\begin{array}{c}
d_{k \mid k} \\
d_{k+1 \mid k} \\
d_{k+2 \mid k} \\
\vdots \\
d_{k+N-1 \mid k}
\end{array}\right]
$$

and

$$
\mathcal{A}=\left[\begin{array}{c}
I \\
A \\
A^{2} \\
\vdots \\
A^{N}
\end{array}\right], \quad \mathcal{B}=\left[\begin{array}{cccc}
0 & 0 & \cdots & 0 \\
B & 0 & \cdots & 0 \\
A B & B & \cdots & 0 \\
\vdots & \vdots & \ddots & \vdots \\
A^{N-1} B & A^{N-2} B & \cdots & B
\end{array}\right], \quad \mathcal{B}=\left[\begin{array}{cccc}
0 & 0 & \cdots & 0 \\
B_{d} & 0 & \cdots & 0 \\
A B_{d} & B & \cdots & 0 \\
\vdots & \vdots & \ddots & \vdots \\
A^{N-1} B_{d} & A^{N-2} B_{d} & \cdots & B_{d}
\end{array}\right]
$$

$\boldsymbol{Q}=\left[\begin{array}{cc}I_{N} \otimes Q & 0 \\ 0 & P\end{array}\right]$ and $\boldsymbol{R}=I_{N} \otimes R$ where $\otimes$ represents the Kronecker product. See e.g., [17,19]. The state $X_{k}$ and the input $u_{k}$ are constrained within desired limits. Let us assume that the constraints are linear and expressed as:

$$
X_{k} \in \mathbb{X}=\left\{X \mid M_{X} X \leq \mathbf{1}\right\}, \quad u_{k} \in \mathbb{U}=\left\{u \mid M_{u} u \leq \mathbf{1}\right\}
$$

where 1 is a vector of all ones. Let us also consider that the worst-case bounds on $d_{k}$ for the relevant ranges of states and inputs are known and $d_{k} \in \mathbb{D}=\left\{d \mid M_{d} d \leq \mathbf{1}\right\}$.

\section{Online MPC Computations}

The MPC optimization problem minimizing the cost in (5) under relevant constraints can be written as:

$$
\underset{\boldsymbol{u}_{k}}{\operatorname{minimize}} J_{N}(k)
$$

subject to $X_{k \mid k}=X_{k}$ and

$$
\begin{aligned}
X_{k+i \mid k} & \in \mathbb{X}, \quad i=0, \ldots, N-1 \\
u_{k+i \mid k} & \in \mathbb{U}, \quad i=0, \ldots, N-1 \\
X_{k+N \mid k} & \in \mathbb{X}_{N}
\end{aligned}
$$

Here, the last constraint in (7b) is called the terminal constraint and it is added to ensure that the terminal state reaches a certain region which may be necessary to establish stability. If the set $\mathbb{X}_{N}$ is polyhedral and $x_{k}$ and $\boldsymbol{d}_{k}$ are known, problem (7) can be solved as a quadratic programming $(\mathrm{QP})$ problem. At any time $k$, we assume that the state $X(k)$ is completely known through measurement or estimation. However, we do not know the vector $\boldsymbol{d}_{k}$. The nonlinear description of $d_{k}$ in terms of $X_{k}$ and $u_{k}$ is complicated and not readily expressible in an analytical form. However, given the values of the control inputs, we can estimate the values of $d_{k+i \mid k}$ by comparing the successive states with the actual states obtained through aposteriori high-fidelity propagation of the orbit of the member satellite. Let a high-fidelity nonlinear propagation for the given reference orbit and the given epoch be described by

$$
X_{k+1}=\Phi_{k}\left(X_{k}, u_{k}\right)
$$

Now, at each time step, we first assume some realistic value of the disturbance vector and then successively carry out the MPC optimization followed by the update of the disturbance through a comparison with the actual nonlinear predicted state trajectory. 
The resulting MPC scheme is summarized in Algorithm 1 below:

Algorithm 1 - Online MPC algorithm:

At $k=0,1,2, \ldots$

- Measure or estimate the state of the system $x_{k}$

- Initialize $\boldsymbol{d}_{k}$ with $d_{k+i \mid k}=0$ if $k=0$ and with $d_{k+i \mid k}=d_{k+i \mid k-1}$ otherwise.

- Repeat

- Solve problem (7) and obtain the optimal input vector $\boldsymbol{u}_{k}^{*}$.

- $\quad$ Set $X_{k \mid k}^{a}=X_{k}$.

- $\quad$ For $i=0$ to $N-1$, find

$$
\begin{aligned}
& X_{k+i+1 \mid k}^{a}=\Phi_{k+i}\left(X_{k+i \mid k}^{a}, u_{k+i \mid k}^{*}\right) \\
& e_{k+i \mid k}=X_{k+i+1 \mid k}^{a}-A X_{k+i \mid k}^{a}-B u_{k+i \mid k}^{*}-B_{d} d_{k+i \mid k}
\end{aligned}
$$

End For

- Set $\overline{\boldsymbol{d}}_{k}=\boldsymbol{d}_{k}$

- $\quad$ Set $\boldsymbol{d}_{k}=\alpha \boldsymbol{d}_{k}+\boldsymbol{e}_{k+i \mid k}$

- $\quad$ Until $\left\|\boldsymbol{d}_{k}-\overline{\boldsymbol{d}}_{k}\right\| \geq T O L$

- $\quad$ Choose and apply the input $u_{k}^{*}=u_{k \mid k}^{*}$.

In Algorithm 1, after each solution of problem (7) in the beginning of the third step, the actual predicted state trajectory based on the high-accuracy nonlinear propagation is obtained. The disturbance vector is then updated based on the comparison with the actual state trajectory. In the last step of the Repeat-Until block, the value of $\alpha$ should be chosen so that the mapping is contractive, and the disturbance sequence converges while the error sequence decays to zero. In most cases, $\alpha=1$ may be the trivial choice that works.

Remark 1: Note that the solution obtained with Algorithm 1 is suboptimal to some extent. Here, we assume that the disturbance vector is a constant external disturbance when, in reality, it may be mainly dependent on the state and the input trajectory of the system. However, for small disturbances such as in the case of the satellite problem, the loss of optimality may be insignificant.

\section{Stability Analysis}

Algorithm 1 can be guaranteed to be stabilizing under certain conditions. For brevity, assume that $\bar{X}_{k}=0$ and $\bar{u}_{k}=0$ for all $k \geq 0$, which means we wish to regulate the state to the origin. Let $K$ be a static feedback gain for (3) and $\mathbb{X}_{N}$ be a convex set such that it is both invariant and constraint-admissible for the closed-loop system under feedback $u_{k}=K x_{k}$. That is, it satisfies the conditions:

$$
(A+B K) \mathbb{X}_{N} \oplus B_{d} \mathbb{D} \subseteq \mathbb{X}_{N}
$$




$$
K \mathbb{X}_{N} \subseteq \mathbb{U}
$$

where $\oplus$ denotes set addition. Further, let the matrix $P$ be the solution of the Lyapunov equation

$$
P-(A+B K)^{T} P(A+B K)-\left(Q+K^{T} R K\right)=0
$$

Theorem 1: Let $K, P$ and $\mathbb{X}_{N}$ be the terminal feedback gain, cost matrix and the terminal constraint set such that the conditions in (9) and (10) are satisfied. Then, if the disturbance sequence converges at every step in Algorithm 1 and that the MPC problem is feasible at time $k=0$, the MPC problem always remains feasible, the state and input constraints are always satisfied and that the state of the system $X_{k}$ is driven into the smallest disturbance invariant set $\mathcal{X}$ satisfying $(A+B K) \mathcal{X} \oplus$ $B_{d} \mathbb{D} \subseteq \mathcal{X}$ as $k \rightarrow \infty$.

Proof: The fact that the problem remains feasible and that the constraints are satisfied at all times under the stated conditions is obvious. The fact that the state is driven into the smallest set satisfying the given disturbance invariance condition can be established by using the standard arguments as in $[17,20]$.

\section{SATELLITE STATE CONTROL USING SUCCESSIVE-OPTIMIZATION-BASED SIMPLIFIED MPC}

As mentioned in the second section, there are several cluster patterns that are naturally supported by orbital dynamics. We consider the case where member satellites are to be initialized or reconfigured into a projected circular pattern of radius 1 $\mathrm{km}$ about a reference point where the orbit of the reference point is specified by the set $\Xi=\left(6850 \mathrm{~km}, 0.01,25^{\circ}, 0,0,0\right)$. We analyze the case of the first satellite whose actual initial states and desired states are shown in Table 1.

Table 1: Actual and Desired Initial States of the Chosen Member Satellite

\begin{tabular}{|l|l|l|l|l|l|l|}
\hline & $\boldsymbol{x}(\mathbf{k m})$ & $\boldsymbol{y}(\mathbf{k m})$ & $\mathbf{z}(\mathbf{k m})$ & $\dot{\boldsymbol{x}}(\mathbf{m} / \mathbf{s})$ & $\dot{\boldsymbol{y}}(\mathbf{m} / \mathbf{s})$ & $\dot{\boldsymbol{z}}(\mathbf{m} / \mathbf{s})$ \\
\hline Actual state & 0.135 & -1.086 & 0.257 & -0.472 & -0.262 & -0.941 \\
\hline Desired state & 0.433 & -0.500 & 0.866 & -0.278 & -0.964 & -0.556 \\
\hline
\end{tabular}

Note that the desired state trajectory beyond the initial state is obtained by the propagation of the initial desired state with the same system matrix $A$ but without any control or disturbance input. We choose to express relative positions in km, velocities in $\mathrm{m} / \mathrm{s}$ and accelerations in $\mathrm{mm} / \mathrm{s}^{2}$ so that the state equation is better scaled. We consider $T=2$ minutes and a total simulation time of 200 minutes. Control input is applied during the first $1 / 6$ th of $T$. In the MPC cost function, we choose

$=\left[\begin{array}{cc}I_{3} & 0 \\ 0 & 0.1 I_{3}\end{array}\right], R=0.001 I_{3}$, where $I_{3}$ is the identity matrix of order 3 . For the terminal cost, we obtain the LQ optimal gain $K$ and the corresponding solution $P$ of the algebraic Riccati equation. We use corresponding disturbance invariant set as the terminal constraint set.

In the simulations, for the exact computation of the predicted satellite trajectory, we use the high-fidelity nonlinear propagator in the spacecraft control toolbox where effects of $J_{2}$, sun-moon influences, atmospheric drag and so on are included. In the MPC algorithm, we consider a horizon length of $N=50$ steps in the beginning. However, as the control progresses, we decrease the horizon length and as the satellite enters the long-term pattern maintenance phase, we use a small horizon length $\bar{N}$.

Fig. 1 shows convergence towards 0 of the norm of the stacked prediction errors during the successive optimization in the first time step $(k=0)$. Based on the nature of this convergence, it is obvious that it is sufficient to repeat the optimizations for about 3 times every time step. Fig. 2 shows the components of position and velocity deviations from the 
desired values when the satellite state is controlled using the scheme of Algorithm 1. The corresponding input components are shown in Fig. 3. Note that input components are computed as accelerations.

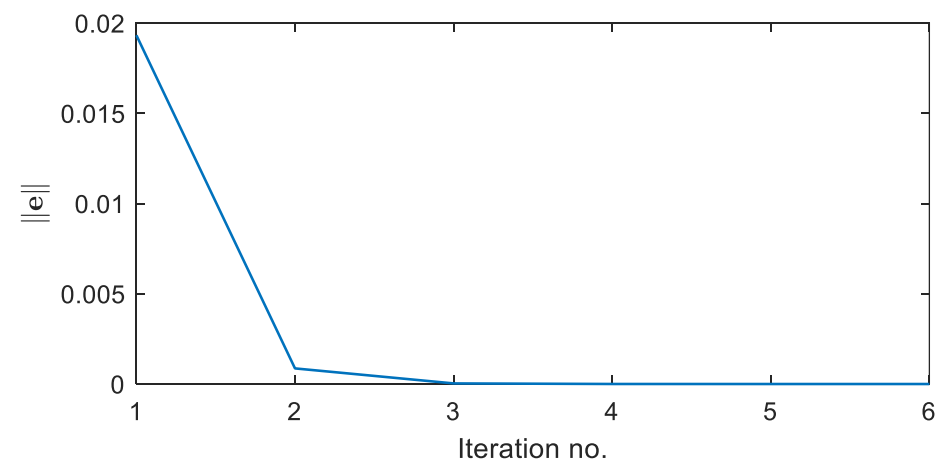

Figure 1: Convergence of the Norm of Stacked Errors defined in Algorithm 1 during Successive Optimization at $k=0$
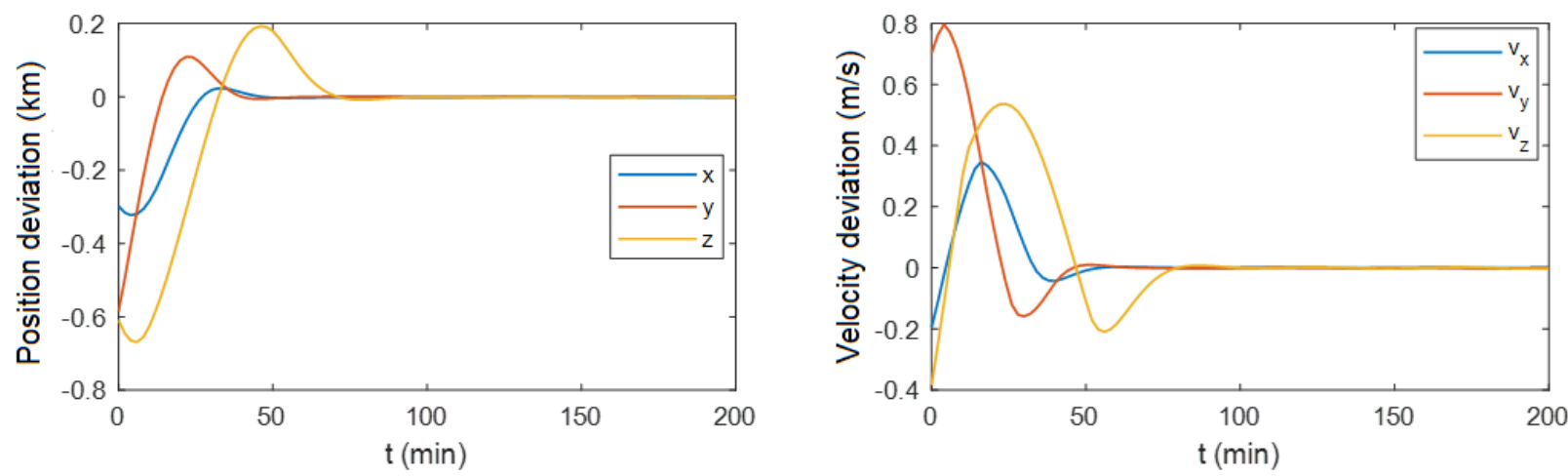

Figure 2: Components of Position and Velocity Deviations from Desired Values
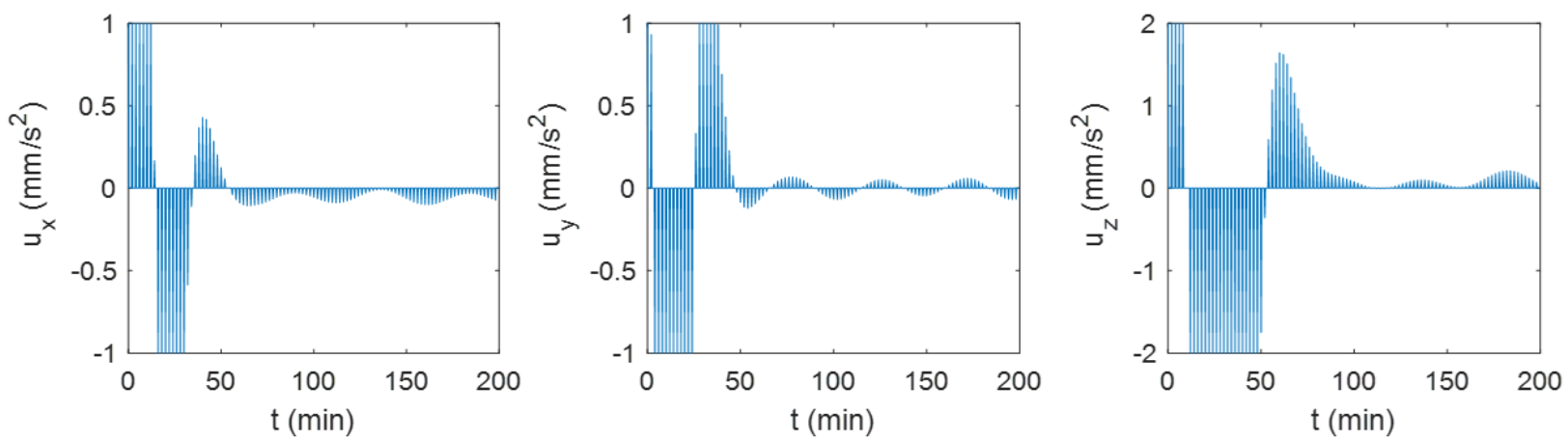

Figure 3: Components of the Control Input.

We wish to compare the performance of the MPC scheme of Algorithm 1 with that of the standard MPC. The former makes computations with more accurate predictions. This is reflected in Fig. 4 where we compare the position errors (deviations from desired values) during the long-term pattern maintenance phase (after $k=50$ ). The dotted lines are the deviations with Algorithm 1 whereas the solid lines are those with the standard MPC. The deviations in this phase depend on various factors such as the cost matrices, horizon length and the terminal constraint. With accurate predictions, it is possible to impose and satisfy tighter constraints if required. The plots in Fig. 4(a) are obtained for $\bar{N}=10$ and those in Fig. 4(b) correspond to the case with $\bar{N}=5$ - the terminal constraint being the same as before. As can be seen from the figures, the deviations with nonlinear MPC are about 50\% lower to more than an order of magnitude less. 
Note that the smaller deviations with the nonlinear MPC have not been achieved with the use of more control efforts. Table 2 lists the $\Delta V$ 's used with the two control techniques in total and in the pattern- maintenance phase (after $k=50)$. It can be seen that $\Delta V$ 's used with the MPC scheme of Algorithm 1 is lower in total and lower by about $30 \%$ in the patternmaintenance phase.

Algorithm 1 is computationally more intensive than the standard algorithm since it needs to repeat the optimization a few times. Further, it also needs nonlinear propagation of the satellite trajectory after every optimization step. Simulations using the qpOASES QP solver in MATLAB in a Windows machine with 16 GB RAM and i7 1.8 GHz processor showed that the solution time for the MPC problem with a horizon length $N=50$ was on the average about $0.08 \mathrm{~s}$ and about $0.5 \mathrm{~s}$ in the worst case, and the nonlinear propagation time was about $0.1 \mathrm{~s}$ on the average and about $0.4 \mathrm{~s}$ in the worst case. Clearly, computations with the standard MPC are faster by a factor of about 5 but the total computation time each time step is just a tiny fraction $T$.

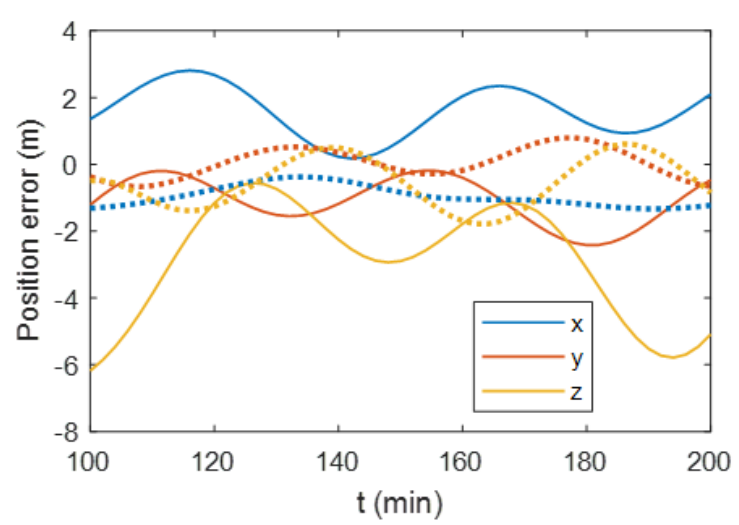

(a)

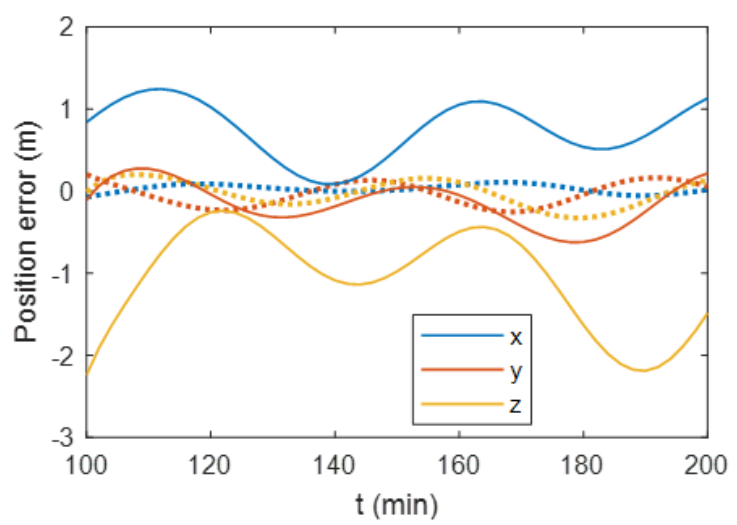

(b)

Figure 4: Position Deviations from Desired Values during Long-Term Pattern Maintenance Phase (a) with $\bar{N}=10$, and (b) with $\bar{N}=5$. The Dotted Lines correspond to the MPC Scheme of Algorithm 1 and the Solid Lines correspond to the Standard MPC

Table 2: $\Delta-V^{\prime}$ 's Applied in the Three Directions

\begin{tabular}{|c|c|c|c|c|}
\hline \multirow{2}{*}{$\Delta \mathbf{V}$} & \multicolumn{2}{|c|}{ Standard MPC } & \multicolumn{2}{c|}{ MPC of Algorithm 1 } \\
\cline { 2 - 5 } & Total & After $\boldsymbol{k}=\mathbf{5 0}$ & Total & After $\boldsymbol{k}=\mathbf{5 0}$ \\
\hline$\Delta \mathrm{V}_{\mathrm{x}}(\mathrm{mm} / \mathrm{s})$ & 550 & 119 & 450 & 58 \\
\hline$\Delta \mathrm{V}_{\mathrm{y}}(\mathrm{mm} / \mathrm{s})$ & 490 & 42 & 480 & 39 \\
\hline$\Delta \mathrm{V}_{z}(\mathrm{~mm} / \mathrm{s})$ & 1403 & 89 & 1350 & 82 \\
\hline
\end{tabular}

\section{CONCLUSIONS}

A simplified nonlinear MPC approach was explored for the precise control of the translational state of a member satellite in a fleet of small satellites. The approach involves the calculation of an optimal control sequence based on a high-precision prediction of the satellite trajectory using successive optimizations interspaced with nonlinear trajectory evaluation and the subsequent update of a disturbance vector representing the nonlinear effects. The performance of the proposed approach was evaluated with high-fidelity simulations and its effectiveness was illustrated. 


\section{ACKNOWLEDGEMENTS}

This paper was supported by Education and Research promotion program of KOREATECH in 2019.

\section{REFERENCES}

1. R.M. Millan, R. von Steiger et al., Small satellites for space science: A COSPAR scientific roadmap, Advances in Space Research, 64(8), pp. 1466-1517, 2019.

2. H. Sanchez et al., Starling 1: Swarm Technology Demonstration, 32nd Annual AIAA/USU Conference on Small Satellites, Logan, UT, August 2018.

3. T.J. Sabaka, L. Tøffner-Clausen, N. Olsen, and C.C. Finlay, A comprehensive model of Earth's magnetic field determined from 4 years of Swarm satellite observations, Earth Planets Space, 70(1):130, 2018.

4. L. Zhou, J-J Luo, T. Nogueira, and K. Schilling, Orbit design and control method for satellite clusters and its applications to NetSat project, Proceedings of the Institution of Mechanical Engineers Part G Journal of Aerospace Engineering, 232(8), pp. 1559-1570, 2018.

5. E. Edlerman and P. Gurfil, Cluster-Keeping Algorithms for the Satellite Swarm Sensor Network Project, Journal of Spacecraft and Rockets, 56(3), 2019

6. D. P. Scharf, F. Y. Hadaegh, and S. R. Ploen. A survey of spacecraft formation flying guidance and control (Part II): Control, Proceedings of the American Control Conference, pp. 2976-2985, 2004.

7. F. Tarek, T. Mahmoud and A.Y. ELRaffieic, Satellite swarm survey and new conceptual design for Earth observation applications, The Egyptian Journal of Remote Sensing and Space Science, In press.

8. J.H. Wang, J.X. Zhang and X.B. Cao, Optimal satellite formation reconfiguration strategy based on relative orbital elements. Acta Astronautica 76, pp. 99-114, 2012.

9. K. Schilling, Networked control of cooperating distributed pico-satellites, IFAC Proceedings Volumes, 47(3), pp.7960-7964, 2014

10. H. Zhang and P. Gurfil, Distributed Control for Satellite Cluster Flight Under Different Communication Topologies, Journal of Guidance, Control, and Dynamics, 39(3), pp. 617-627, 2016

11. C. Sabol, R. Burns, and C. A. McLaughlin. Satellite formation flying design and evolution. Journal of Spacecraft and Rockets, 38(2), pp. 270-278, 2002.

12. Y. Menard, R. Bock, E. Neri, and R. Haagmans, Swarm Mission Concept, Proceedings of the First Swarm International Science Meeting, Nantes, France, May, 2006.

13. L. Breger, I. Gokhan, M. Tillerson, and J.P. How, Cooperative spacecraft formation flying: Model predictive control with openand closed-loop robustness. Modern Astrodynamics 1, Elsevier Astrodynamics Series, pages 237-277, 2006.

14. A. Gautam, Y.-C. Chu, and Y.C. Soh, Robust Predictive Control of Satellite Formations, Proceedings of 10th Intl. Conf. on Control, Automation, Robotics and Vision, Hanoi, Vietnam, 2008.

15. S. Di Cairano, H. Park, and I. Kolmanovsky. Model predictive control approach for guidance of spacecraft rendezvous and proximity maneuvering, International Journal of Robust and Nonlinear Control, 22, pp. 1398-1427, 2012.

16. P.A. Felisiak, K.S. Sibilski, K. Qin, G. Li, and W.A. Wróblewski, Nonlinear model predictive control of spacecraft relative motion, Proceedings of the Institution of Mechanical Engineers, Part G: Journal of Aerospace Engineering, 233(11), pp. 39063919, 2019. 
17. A. Gautam, Y.-C. Chu, and Y. C. Soh, Optimized dynamic policy for receding horizon control of linear time-varying systems with bounded disturbances, IEEE Transactions on Automatic Control, 57(4), pp. 973-988, 2012.

18. T. Petero, E. Hemandes, Y. Arkun, and F.J. Schork, Nonlinear predictive control of a semi-batch polymerization reactor by an extended DMC, Proceedings of the American Control Conference, pp 1534-1539, 1989.

19. J.M. Maciejowski, Predictive Control with Constraints, Prentice Hall, Harlow, England, 2002.

20. L. Chisci, J. A. Rossiter, and G. Zappa. Systems with persistent disturbances: predictive control with restricted constraints. Automatica, 37, pp. 1019-1028, 2001. 

